\title{
Feature Use in Mobile Video Creation
}

\author{
Arto Puikkonen $^{1}$, Jonna Häkkilä ${ }^{2}$, Rafael Ballagas ${ }^{3}$, and Jani Mäntyjärvi ${ }^{4}$ \\ ${ }^{1}$ Nokia Research Center, Visiokatu 1, \\ 33720 Tampere, Finland \\ ${ }^{2}$ Nokia Research Center, Yrttipellontie 1, \\ 90401 Oulu, Finland \\ ${ }^{3}$ Nokia Research Center, 955 Page Mill Road, \\ 94304-1013 Palo Alto, USA \\ ${ }^{4}$ VTT, Kaitoväylä 1 , \\ 90570 Oulu, Finland \\ \{arto.puikkonen, tico.ballagas, jonna.hakkila\}@nokia.com, \\ jani.mantyjarvi@vtt.fi
}

\begin{abstract}
Today's mobile phones are also video cameras. People are using these ubiquitous cameras to document everyday surroundings as well as create more artistic videos. This paper examines emergent mobile film making patterns by tracking video composition and recording activities in ecologically valid contexts of use. We report the findings of a user study on user created mobile videos, where the actions of 11 active mobile video users were documented for 2 weeks. The collected material included diaries, device logs, and altogether 255 videos. Our findings characterize the features of a typical mobile video. Additionally, our study uncovers common practices, user motivations and pitfalls during filming and editing in the mobile context.
\end{abstract}

Keywords: Mobile video, mobile multimedia, user created content, user studies.

\section{Introduction}

Video has become an integral part of the way we capture, and share experiences. The emergence of popular online video sharing websites has significantly lowered the threshold for distributing video content, and people are now able to capture video in more circumstances than ever before and share it instantaneously. Earlier research reveals that the lightweight video work, meaning the use of mobile phone for shooting the videos, included more spontaneous and 'just for fun' filming in comparison to the use of conventional video cameras [3]. Other studies related to mobile videos include collaborative live video creation [1] and the practices on consuming videos [4] or mobile TV content [2], where for instance gift giving practices (e.g. recording content for a friend) were exposed [2]. To the authors' knowledge, this is the first study focusing on charting the technical and editing features used in everyday practices with user created mobile phones videos. Due the limited length, this paper has concentrated on the structure and feature use findings. In their further work, the authors will seek to look deeper into the context and content side of mobile phone video creation. 


\section{Study Setting and Resulting Videos}

For a study in summer 2008, a total of 12 participants (10 male, 2 female) were recruited with a variety of occupations and all from Finland for a study. The average age was 30 years, from 16 to 45 . One of the participants, \#10, dropped out during the study. Each participant received a Nokia N95 mobile phone for 2 weeks, which includes a camera for video. The phones were equipped with key logging software, which gathered all key presses with a time stamp within the camera, gallery and editor application. For the 2 weeks, the participants were asked to use the given mobile phone just as they use their own. The participants were also provided with a small paper diary about filming and transferring, which the participants were asked to fill in whenever such an event occurred. In the end, each participant was interviewed. All the videos were analyzed for content, context, structure and feature use. This analysis was supported by analyzing the logger, interview and diary data.

The participants filmed a total of 266 videos with the provided mobile phones, resulting in the average of 24.2 videos, minimum being 4 and maximum 90 clips. Three $(\# 1,4,11)$ removed 11 videos during the study. In addition, 10 videos were accidental extremely short ones, making the total amount of analyzed videos 245. (See Fig. 1)
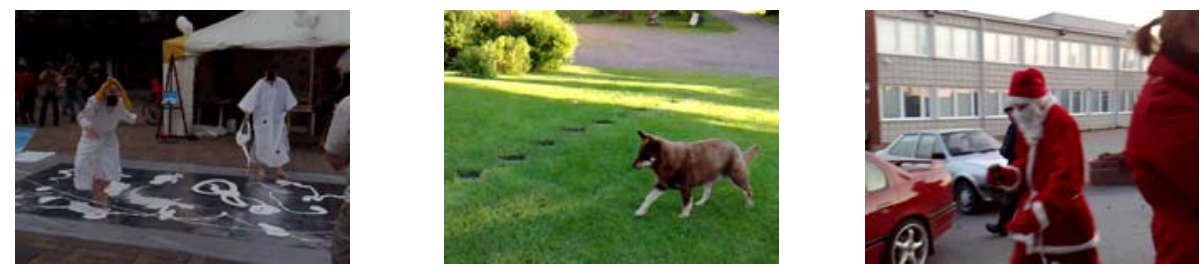

Fig. 1. Example screenshots from user created videos

\section{Feature Use Related to Filming Mobile Videos}

A vast amount of videos, (222/245), were single clip. Only two participants created videos with multiple clips $(\# 9,11)$. Out of the 42 videos by user \#9, 17 had multiple clips and for user \#11, 6/28 had multiple clips. The average amount of clips for each multiclip video was 2.6 varying from 2 to 4 clips, 3 being the most frequent. With clip length in multiclip videos, the average length was 29.5 seconds.

Interestingly, all multiclip videos were created by using the phones provided pause function, which enables you to halt and resume filming when desired. When asked in the end interview also users \#5 and \#7 stated that they sometimes use this function. The motivation is getting a rough editing with little effort, as further editing was unlikely. "I just want to cut out stuff so it makes it easier to edit it later or that it is already edited as I probably won't have the energy to edit it later on." (\#7). It was stated that this kind of editing had its downsides, but is most often used due to its level of ease. "It leaves extra bits in it." (\#7)

The other 7 participants stated that they do not use this feature. Reasons for not creating multiclip videos divide into two categories. Five of the users expressed that they want the videos to be single entities as the videos are consumed as single entities. 
"Do not need it as they are for single items." (\#6) For the rest, the reason is the unexpected nature of the filmed target, which then would require more user attention.

Besides the pause feature, zoom typically is the easiest feature to access in mobile phones. Not surprisingly, 8 of the participants used zoom during the study and also 1 other stated using zoom occasionally. In total, zoom was used with 71 videos out of the 234 videos they created altogether. But commonly among all participants the opinion about the zoom was negative, and considered mainly as 'a necessary evil'.

Zooming was more preferred during filming instead of before filming. Out of 71 videos with zoom, 70\% (50) videos had zooming during filming. 65\% (46) had forward zooming and 55\% (39) videos backward zooming. 49\% (35/71) of the videos included zooming prior to filming. During interviews and by analyzing the videos, it became clear that people were less rushed in these circumstances and thus had more time to spend on video composition.

A significant factor discouraging the use of zoom is the perceived decrease in video quality. Many of the videos are eventually consumed on larger screens where the downside of reduced quality is very apparent. Even for those that consume video on mobile phones, the use of zoom is seen as very unpleasant as the decrease in quality is apparent when filming. These issues make the use of zoom even more unpleasant for many and limits the use to situations where it is very necessary.

When probed about the situations where zoom was necessary, the response was distance to the target. In these situations, it is either impossible to get closer, getting closer would have an undesired impact on the target, or getting closer would be socially awkward. In addition, two users reported that using the zoom is an easy way to get some life into the video, if the target itself is not lively enough.

In general, when examining the actions done during filming, the remarkable observation was the lack of them. During the study, none of the users changed any video settings of the device that are available through the menus in the UI. Even for video resolution, the common consensus was that it is initially changed to the desired quality and left that way for good. When asked about the use of the other settings, no user changed from the default. Reasons for using default settings divide into two categories. For some, the default settings for white balance and color tone are seen as the best alternative in almost every situation. For others, the reason is simply not knowing what setting would be the best or even what the effect of these settings is. Thus the defaults are the "easy way out" for a good quality end-result.

As every day situations often have a sporadic nature, changing the settings before filming is out of the question if it delays capture. The only situations, where settings are changed, are nighttime situations when being fast is not required. This event did not occur during the study, but was found during interviews $(\# 4,6)$. For both the night mode feature provided needed improvement, when the received quality is generally poor. However, for both, avoiding filming in poor lighting was still the preferred option.

Many of today's mobile phones offer editing capabilities, such as the possibility to merge videos, give the file a name, and replace audio. Similarly as none of the multiclip videos during the study were done by using the merging feature, the use of other features after the filming was also minimal. The logger data showed that no-one had used the editor, none of the videos had been given names and none had changed the audio using the provided features. When this behavior was presented to participants, it was clear that this also represented their practices in every day life. 
Out of the 11 , only $3(\# 5,11,12)$ stated that they had ever tried to use a video editor on a mobile phone. Out of the 3 that have tried the editor, none have used it more than a few times. For all three, the experience was seen as slow, cramped controls and requiring too much effort. "You can't do stuff like that with a small screen when even the PC screen sometimes feels too small for editing." (\#11) All three that have tried the editor provided by a mobile phone actively utilize an editor provided by a PC.

For the 7 participants that have not even tried to use the editor, the reasoning can be divided into two categories. The $4(\# 2,3,7,8)$ that have tried and used editors on a PC stated that the mere idea of editing on a mobile phone is seen as too complicated. This level of complication is compared to their experiences with PC editing that is also seen as challenging. The $3(\# 1,4,6)$ that haven't tried editing on any device stated that there is no need to edit on a mobile as there is no need to edit on a PC either. "I really don't see it as important as I don't need editing." (\#1) The reasons for not seeing the editing as important for the three were "My videos are not movie-like, only documents of events." (\#1), "I don't have time for that." (\#4) and "They are just individual clips." (\#6)

When asked about the possibility to give names to video files, none of the users were interested in the feature. In fact, none of the users give names to the files at any point of the video life, until it is a complete edited entity. When it is an edited complete version, users 5 and 12, who actively edit videos, sometimes give the end versions names. Naming files is typically done where editing is done, which is on a PC. During the study user 12 created one edited complete version and gave that a name. The name of the file was the name of the lake where the video was filmed.

As a conclusion, mobile phone videos form an important part in capturing a variety of everyday events. As the current solutions may be fairly suitable for some users, the more active users clearly experience technical limitations when using a mobile phone to capture the things that surround them.

\section{References}

1. Engström, A., Esbjörnsson, M., Juhlin, O.: Mobile Collaborative Live Video Mixing. In: Proceedings of MobileHCI 2008, pp. 157-166. ACM Press, New York (2008)

2. Harper, R., Regan, T., Izadi, S., Mosawi, K.A., Rouncefield, M., Rubens, S.: Trafficking: design for the viral exchange of TV content on mobile phones. In: In Proc.of MobileHCI 2000, vol. 309, pp. 249-256. ACM, New York (2000)

3. Kirk, D., Sellen, A., Harper, R., Wood, K.: Understanding Videowork. In: Proc. CHI 2007, pp. 61-70 (2007)

4. O’Hara, K., Mitchell, A.S., Vorbau, A.: Consuming Video on Mobile Devices. In: Proc. CHI 2007, pp. 857-866 (2007) 